\title{
Fauna insectorum Helvetiae.
}

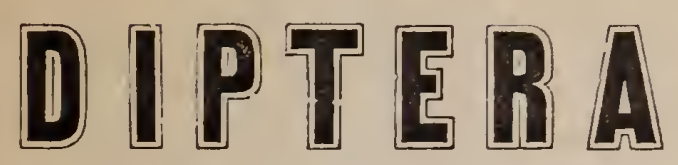

Die Familien der Fliegen und die Genera und Species der Waffenfliegen, Bremsen, Schwebfliegen und Raubfliegen.

Analytisch zusammengestellt

von

Dr. Gustar Schoch.

Sichafihanseu

buchdruckerei von Bolli \& böcherer $18: 10$. 



\section{Einleitung.}

Die Dipteren sind die Vögel unter den Insekten, nicht nur wegen ihres vollendeten Flugvermögens, sondern mehr noch wegen der Monotonie ihres Baues. So wenig man einen Vogel mit irgend einer andern Thierform verwechseln kann, so wenig wird man die Fliege oder Mücke verkennen, und die wenig zahlreichen Fälle, wo Fliegen Wespen und Bienen nachahmen, werden sofort aufgeklärt, sobald man die zwei Flügel genauer betrachtet. In so monotonen und artenreichen Ordnungen ist dann aber die Unterscheidung der Familien, Gattungen und Species um so schwerer, wir müssen daher zur allgemeinen Orientirung etwas näher in die Criterien eintreten, die systematisch wichtig sind. Bei dieser Gelegenheit soll hier ausdrücklich betont werden, dass nur diejenigen Momente hervorgehoben werden, die zum leichten Bestimmen dienen, nicht aber biologisch und physiologisch interessante Terhältnisse. Für diese verweisen wir auf die dipterologischen Hauptwerke von Meigen, Schiener, Loew und anderen. Um Abbildungen zu umgehen, ersuchen wir denjenigen, der sich noch nie näher mit Dipteren beschäftigt hat, einige grössere gemeine Fliegen und Mücken auf die hier beschriebenen Organe mit der Lupe zu prüfen. Zum Studium des Flügelgeäder's ist es passend, einigen verschiedenartigen Dipteren die F'lügel auszurupfen, zwischen 2 Glasscherben zu legen und das relativ einfache Geäder stark vergrössert abzuzeichuen.

Der Kopf der Dipteren ist durch die meist grossen Augen kugelig. Die Fühler sind bei den Fliegen aus drei ungleichen Gliedern gebildet, deren letztes zuweilen geringelt oder mit einer Borste oder einem griffelartigen Anhang versehen ist. Bei den Mücken sind sie 6- oder mehrgliederig, die einzelnen Glieder unter sich gleich. Die stark vorgequollenen Fascetaugen sind bei den Mänuchen meist grö̈sser als bei den Weibchen, sodass sie an der Stirne einander oft berühren. Sie haben gleich oder ungleich grosse Fasceten, indem bei einigen Tabaniden z. B. die Fasceten der einen Augenhälfte grösser sind als die der andern. Oft sind sie im Leben schön gefärbt, mit purpurrothen oder grünen und schwarzen Binden geziert, die nach dem Austrocknen schwer zu erkennen sind. Ob sie nackt oder fein behaart sind, erkennt man nur mit guter Lupe unter schiefer Be- 
leuchtung. Die meisten Dipteren haben anf der Stimpartie 3 Punktangen. Der Ranm hinter und über den Angen heisst Scheitel, der zwischen den Augen Stirn, der unter denselben Gesicht oder Untergesicht. Das Gesicht ist, von der Seite betrachtet, oft concav ausgehöhli, nnd verschiedenartig behaart und beborstet. Man unterscheidet den Kinebelbart (= Mystax), der in der Mitte des Gesichtes hinzieht und oft höckerige Auftreibungen zeigt, und den Backenbart (= Barba), der die Seitenpartien nnter dell Augen, die sog. Wangen (genac) bedeckt. Am nntersten Ende des Gesichtes liegt der Mund mit den Fresswerkzeugen. Das Hauptorgan derselben ist der Rüssel (Unterlippe = Labimm), eine oft gekniete Hohlsonde oder gespaltene Röhre mit 2 Tasteru (palpi). Sie dient zur Aufnahne der durchweg flüssigen Nahrung, ist also ein Saugapparat. Als stechende Organe kommen hierzu 2 oder 4 hohle Stechborsten, d. h. ein oder zwei Paar metamorphosirte Kiefer (Oberkiefer- und Unterkieferborsten). Indess können auch alle stechenden Elemente felılen. Zn den 4 Kieferborsten treten bei einigen Familien noch 2 andere stechende Stücke, eine lanzetlich rerlängerte Oberlippe (labrum) nnd ein chitinisirter Anhang der Unterlippe oder des Rüssels, die Zunge, so dass im ganzen 6 borstenartige Stücke vorhanden sein können. Wir werden übrigens in den folgenden Tabellen diese sehr kleinen Theile so wenig als möglich verwenden.

Der 'Thorax besteht aus drei fest mit einander verwachsenen Segmenten und ist der Träger der Beine und Flügel. Die Beine der Fliegen sind kräftiger, die der Jücken sehr zart gebant, verschiedenartig behart und bedornt, init 5 Fnssgliedern. Die Füsse tragen oft, aber nicht inmer, 2, seltener 3 Haftläppchen zwischen den Klanen. Die Flïgel sind bei den Mücken meist gestielt oder allmälig gegen die Basis verengert, bei den Fliegen etwas erweitert mit emer lappenarigen basalen Einschnïrung, dem Flügelläppehen, versehen. Hinter nud unter ihnen liegt das Flïgelsch üppchen, ein zartes rom Flïgel getrenntes Häntchen, eine Art rudimentärer Hinterflügel, der aber anch ganz fehlon kann. Am letzten Thoracalsegment endlich bemerkt man zwei geknöpfte Stäbchen, die Schwingkolben oder Halteres, deren Farbe oft characteristisch ist.

Was nun das Flïgelgeäder anbelangt, so bietet es die wichtigsten Anhaltspnnkte für die Systematik nnd muss dalıer specieller besprochen werden. Wir schliessen uns biebei der unverfänglichen Nomenclatur an, die Schiner in seiner Fauna dipterorum anstriaca anwendet, weil dieselbe Bezeiohmungsweise von IIrn. Prof. Huguenin in der Behandlung der Tipuliden eingeführt worden ist (Mittheilungen der Schweizer. entomol. Ge- 
sellschaft). Wir zählen demuach die Längsadern von vorn nach hinten, indem wir die den Vorderrand des Flügels stützende und umziehende Costalader ausser Betracht lassen. Die Längs a de rn sind:

Costalis, Vorderrandader, bildet den Vorderrand und zieht sich oft, wenn auch verdünnt, um den ganzen Flügel bis zum Flügellappen herum, den Rand bildend.

I. Ader, Subcosta, Unterrandader, ist die erste Ader in der Flügelfäche und mündet stets in den Vorderrand aus. Sie ist immer einfach, selten mit der Costalis verschmolzen, in welchem Falle sie eben als fehlend bezeichnet wird. (Andere geben der Subcosta den wenig bezeichnenden Namen Mediastina.)

II. Ader, Radius, (Subcosta autorum). Geht entweder isolirt oder mit der Subcosta verbunden aus der Flügelwurzel heraus und endet unverzweigt vor der Flügelspitze im Vorderrand. Wo die Subcosta fehlt, ist der Radius die erste dicke Längsader, die den Vorderrand der Flügelmembran stü̈zt, in einigen Fällen läuft sie gemeinsam mit der Subsosta verschmolzen und tritt nur als kleinel Hinterast derselben zum vordern Flügelrand.

III. Ader, Sector-Radii (Radialader autorum). Der Sector ist ein vom Radius um seine Witte herum sich abzweigender Lüngsast, der meist gegabelt, hinter der Flügelspitze in den Rand eimmündet. Es ist die letzte Ader des Radialgebietes, die den kräftigern Vorderrand des Flügels zu stützen hat. Zwischen dieser dritten und vierten Ader ist fast konstant eine deutliche Querader, die normale oder kleine Querader, welche die Grenze des radialen und ulnaren Gebietes scharf markirt.

IV., V. und TL. Lïngsader. Culital- oder Ulnaradern. Zur Stützung der Mitte und hintern Partie der Flügelmembran treten mun meist drei stärkere Aeste aus der Flügelbasis aus und gehen in den ILinterrand. Heistens kommen sie mit gemeinsamem Stamm, seltener isolirt aus der Wurzel und bilden dalıer ein gut erkenntliches, zusammen gehörendes Adersystem.

$I \mathrm{~V}$. Ader, Cubitus anticus = unaris primus (Discoidalader der Autoren.)

V. Ader, Cubitus medius = ulnaris secundus (posticatis autorum), meist der kräftigste der drei Aeste.

VI. Ader-Cubitus posticus = uluaris tertius (analis autorum). Je nachdem diese Adern einfach oder gegabelt in den Flügelhinterrand münden, grenzen sie au demselben eine Anzahl Hinterrandzellen ab, deren erste zwischen sector Radii und cubitus anticus liegt, also ansserhalb der kleinen Querader, die zweite zwischen cubitus anticus und medius etc. 
T'II. Ader, Axillaris. Oft geht noch ein kleines destchen von der Flügelwurzel direct in den hintersten Randtheil, das wir Axillarader nennen wollen.

Zur Orientirung suche man immer zuerst die normale, kleine oder vordere Querader. Vor ihr liegen 3 Adern und hinter ihr 3. Die 3 vordern Längsadern sind Ader I., II. und III., oder subcosta, radius und scctor, die 3 hintern Längsadern sind $\mathrm{Nr}$. 4, 5, 6 oder cubitus primus, secundus und tertius. Hat es vor der normalen Querader nur zwei oder einen Stamm, so sind ebcn Verschmelzungen vorhanden, und sind linter ihr 4 Längsadern bemerkbar, von denen die letzte direct aus der Flügelwurzel tritt, so haben wir noch eine Axillaris. Das radiale Gebiet nimmt den Vorderrand des Flügels ein und hat nie Queradern, das cubitale beherrscht die Flügelfläche bis zum Hinterrand und zeigt oft noch einige Quercommisuren.

Queradern.

1. Normale oder kleine Querader zwischen Ader 3 und 4, sehr selten fehlend.

2. II intere Querader. Fine Querader zwischen Ader 4 und 5 , also zwischen cnbicus anticus und medius oder ihren Seitcnästen.

Andere Queradern haben keine characteristische Stellung, schliessen aber sehr gut markirte Zellen ab. Selnr verbreitet ist eine kleine Wurzelquerader, die oft hart an der Wurzel die Längsadern durchsetzt und meist an der costa oder subcosta beginnt.

Zellen. Wir wollen bei Bezeichnung der Räume (areae) und Zellen das Princip befolgen, dieselben in der Hauptsache nach den sie umschliessenden oder von hinten begrcnzenden Längsadern zu benennen. Eine einzige Ausnahme macht die Discoidolzelle, die sofort auffällt, wenn sie geschlossen ist durch eine Querader und leicht zu entdecken sein wird, da die normale Querader auf sie mündet.

1. Area subcostalis, Raum zwischen costa und subeosta (mediastina autorum).

2. Area radialis, zwischen radius und subcosta, fehlt natürlich, wo radius und subcosta oder costa und subcosta verschmolzen sind (Randzelle).

3. Area interradialis. Zwischen radius und sector. Gabelt sich der sector Radii mehrfach, so haben wir eben mehrere areae interradiales (Unterrandzelle). Die normale Querader theilt nun den Raum zwischen radialem und ulnarem Gebiet in zwei Hälften, eine basale und eine apicale, wir erhalten hier also zwei Zellen.

4. Vordere Basalzelle als basales Feld und 
5. Erste Hinterrandzelle als apicales Feld.

6. Discoidalzelle, die stets zwischen den Aesten des cubitus anticus und medius liegt und von einer Querader nach aussen begrenzt wird. Auf dieselbe mündet die normale Querader; sie felrlt, wenn keine Querader den Raum von dem Flügelrand abschliesst.

In diesem Falle mündet der Raum zwischen sector Radii und cubitus anticus offen in den Flügelrand und heisst dann wie alle offenen Randzellen $\mathrm{z}$ weite $\mathrm{Hin}$ terrandzelle.

Dieser folgen zwischen den Aesten und Gabelungen der Cubitaläste die dritte, vierte etc. Hinterrandzelle, d. h. alle offen in den Aussen- oder Hinterrand mündenden Räume vom Ende des radialen Gebietes bis zum cubitus posterior, der 6 . Längsader.

Zwischen cubitus anterior und cubitus medius liegt im basalen Theil die cellula basalis secunda, die $z$ weite oder hintere Basalzelle, sofern dieser Raum durch eine Querader von der Discoidalzelle abgeschlossen ist.

An al zelle heisst der Raum zwichen den Wurzelu des cubitus medius (Ader 5) und posterior (Ader 6). Sie ist bald offen in den Flügelrand führend, bald lang gestreckt aber geschlossen durch Verbindung von Aesten des cubitus medius und posterior (gestielt), bald sehr kurz, durcl eine Querader abgetrennt und gibt gute systematische Anlialtspunkte. Alles was hinter cubitus posterior liegt heist Area ax illaris.

Hinter dem Flügel sitzen die Flügelschüppchen, blasse ungeaderte Häutchen, die bei einigen Fannilien recht stark hervortreteı, bei anderı so verkümmern, dass sie ganz zu fehlen scheinen.

Der Hinterleib hat keine gegliederten Anhängsel, als die männlichen Geschlechtsorgane, die z. B. bei den Raubfliegen oft sehr stark entwickelt sind. Ihre Grösse und Prominenz ist ein bequemes Mittel, gewisse Arten zu trennen, aber auf den Bau dieser complizirten Zangenorgane gehel wir in den folgenden analytischen Tafeln nicht ein.

Eine ganz besondere Berücksichtigung verdient das Haarkleid der Dipteren, ja man hat eine eigene Disciplin aus seinem Studium gemacht, die Chaetotaxie. Wir finden die verschiedensten Stellen des Kopfes, der Beine, des Schildchens und besonders des Hinterleibes oft mit Borsten und Haaren bekleidet und da unterscheidet man dann besonders von der gewöhnlichen Haarbekleidung (pili) jene grössern, steifen Borsten (Macrochaetae), die stets eine ganz characteristische Stellung und Gruppirung am Körper zeigen, und $\mathrm{zu}$ werthvollen diagnostischen Merkmalen werden. Sie überragen die andern Haare bedeutend und sind leicht kenntlich. 
In systematischer Hinsicht hat man neuerdings nach Brauers Vorgang die Entwicklungs-Geschichte herbeigezogen und die Diptera in zwei grosse Unterklassen getheilt, Orthohapha und Cyclorhapha. Die Ersteren haben eine gemeisselte Puppe, bei letztern erstart bei der Verpuppung die Larvenhaut zur Tonnenpuppe, die mit kreisförmigem Deckel aufspringt. Da es uns hier nur um die Determination der Insekten zu thun ist, so behalten wir die alte Eintheilung der Dipteren in kurzhörnige Fliegen, langhörnige Mücken und vivipare Parasiten bei, und schliessen von den Dipteren die Flöhe (pulicidae) ganz aus, da ihr aus drei getrennten Thoracalringeln bestehender Brustkorb und die anders gestalteten Mundwerkzeuge keine engere Verwandtschaft mit den Dipteren verrathen. Im übrigen schliessen wir uns im Allgemeinen an die klassische Fauna austriaca von Schiner, die jeder besitzen wird, der sich näher mit dieser Insektenordnung befassen will, und lassen die einzelnen Familien in beliebiger Folge zur Bearbeitung gelangen, wie es eben das vorhandene Material erlaubt, den Löwenantheil jedenfalls jüngern Kräften und spätern Bearbeitungen zuweisend. -

\section{Die Familien der Fliegen.}

\section{Subordo. Pupipara $=$ Parasitenfliegen.}

- Körper von elastisch hornartiger Consistenz. Der Kopf dem Thorax aufliegend oder in denselben eingesenkt. Mundtheile bestehen aus einem einfachen Stachel mit zwei seitlichen Klappen, ohne Taster. Hüften durch ein breites Bruststück weit getrennt. Hinterleib undeutlich segmentirt, meist flach gedrückt. Klauen sehr stark. Parasiten von warmblütigen Thieren, oft mit reducirten oder ganz verkümmerten Flügeln. Gebären lebende Larven von Madenform, die sich sofort nach ihrer Geburt verpuppen.

- Körper von gewöhnlicher chitiniger Consistenz. Kopf frei am Thorax vorn anhängend. Mund mit Unterlippe und Tastern, mit oder ohne Stechborsten. Hüften einander genähert. Flügel stets entwickelt, nur ein Paar

\section{Subordo. Nematocera = Mücken oder Langhörner.}

1. Körper und Beine zart. Fühler aus zahlreichen (mindestens 6) unter sich gleichen Gliedern bestehend, 
fadenförmig oder gefiedert. Taster 3-4 gliederig. Fliigel meist schmal, gestielt oder gegen die Wurzel allmälig verengert.

\section{Subordo. Brachycera = Fliegen, Kurzhörner.}

- Körper robuster, Beine kürzer und kräftiger gebaut. Fühler aus 3 unter sich ungleichen Gliedern bestehend, deren letztes oft eine Borste oder ein griffelartiges Endorgan trägt. Oft ist das Endglied geringelt und könnte als mehrgliedrig imponiren. Taster 1-3gliedrig. Flügel an der Wurzel zu einem Läppchen erweitert, selten gestielt.

NB. Bei der Analyse der Fliegenfamilien erlauben wir uns, einige ganz kleine Familien zu unterdrücken, indem wir sie den nächst stehenden grössern beifügen, und müssen daher die Diagnose etwas anders fassen, als dies Schiner in seiner analytischen Tabelle thut.

\section{Subordo. Brachycera, Fliegen.}

1. Fühler 2-3gliedrig, hart am Mundrand eingefügt, Hüften verlängert. Hinterschenkel verbreitert und plattgedrückt. Die grossen Flügel haben bloss 2 dicke Längsadern, die nur die Mitte der Flügelsbreite erreichen und sich dann in zarte Aeste aufösen. Queradern fehlen ganz. Kleine Fliegen, die rasch herumrennen, selten fliegen (Hypocera).

Fam. Phoridae, Lauffliegen.

- Fühler dreigliedrig, weit vom Mundrand eingefügt. Geäder auf der Flügelfläche reichlich, verzweigt, mit deutlichen Queradern Drittes Fühlerglied geringelt, scheinbar mehrgliedrig. Dritte Längsader (sector Radii) stets gegabelt. Die Fühler mit oder ohne Endborste oder Griffel, aber nie mit rückenständiger Borste (Cyclocera) . . . 3

- Drittes Fühlerglied nicht geringelt oder segmentirt 4

3. Die den Vorderrand umkreisende Ader reicht nur bis zur Flügelspitze, umzieht den Hinterrand nicht. Schildchen häufig mit Dornen. Geht die Randader auch auf den Hinterrand über, so ist das Schildchen meist bedornt. 1. Fam. Stratiomyidae, Waffenfliegen.

- Die Randader umzieht fast in gleicher Dicke auch den hintern Flügelrand. Schildchen nie mit Dornen bewaffnet.

2. Fam. Tabanidae, Bremsen. 
4. Fühlerborste oder Griffel ganz fehlend, oder wenn vorhanden, immer endständig (Ortlocera) . . . . 5

- Fühlerborste immer vorhanden und stets rückenständig am letzten Fühlerglied (Athericera) . . . 16

5. Flügelschüppchen schuppenförmig, aussergewöhnlich gross. Plumpe, dickbäuchige, kleine Fliegen mit selır kleinem, nach unten hängendem Kopf an dem buckeligen Halsschild, der nur aus den grossen Fascetaugen zu bestelien scheint, Flügel zart und klein; schlechte Flieger. Fam. Acroceridae BuckelHiegen.

- Flügelschüppchen klein und von gewöhnlicher Form 6

6. Stirne und Scheitel zwischen den gross vorquellenden Augen tief eingesattelt. Fam. Asilidae Raublliegen.

- Stirne und Scheitel zwischen den Augen eben oder den obern Augenrand oft überragend, nie eingesattelt

7. Dritte Längsader (sector Radii) gegabelt . . . . 8

- Dritte Längsader nicht gegabelt . . . . . . . 12

8. Aus der Discoidalzelle oder aus dieser und der hinter ihr liegenden hintern Basalzelle gehen höchstens 3 Längsadern zum Flügelrand ab

- Aus der Discoidalzelle oder aus dieser und der hintern Basalzelle gehen mindestens 4 Adern zum Flügelrand $a b$.

9. Drittes Fühlerglied ohne Griffel oder Endborste.

Fam. Scenopinidae, Fensterfliegen.

- Drittes Fiihlerglied mit Borste oder Griffel 10

10. Analzelle (zwischen Ader 5 und 6) bis zum Flügelrand reichend, offen oder geschlossen, im letztern Falle höchstens kurz gestielt. Meist stark behaarte Fliegen mit grossem Kiopf und oft gefleckten oder gefärbten Flügeln, deren Hinterleib kurz oval ist. Leben parasitisch bei Bienen und haben einen eigenthümlich schwebenden Flug.

Fam. Bombylidae, Schwebfliegen.

- Analzelle kurz, geschlossen und lang gestielt, nie zum Flügelrand reichend. Nackte, schlanke, düstere Fliegen mit kleinem Kopf und spitzen Hinterleib. Fam. Empidae (partim), Schnabelfliegen.

11. Drei Haftläppchen am Klauenglied (da das Empodium stark entwickelt ist). Drittes Fühlerglied mit einer Endborste. 
- Jur 2 Haftläppchen. Drittes Fühlerglied mit einem Endgriffel.

Fam. Terevidae, Reiherfliegen.

12. Fliigel rorn in eine Spitze auslaufend, da sich Vorderund Hinterrand in spitzem Winkel begegnen. Keine Querader auf der Flügelmitte.

Fam. Lonchopteridae, Bachfliegen.

- Flügrel vorn mit abgerundeter Spitze . . . . . 13

13. Flügel mit deutlichen Afterläppchen . . . . . 14

- Flügel mit fehlendem oder rudimentärem Läppchen 15

14. Fühler mit Endborste.

Fam. Platypezidae.

- Fühler mit Endgriffel. Hinterleib nach unten eingebogen. Fam. Conopidae (partim), Dickkopffliegen.

15. Vordere Basalzelle (zwischen 3 und 4) durch eine Querader nahe der Flügelwurzel abgegrenzt, daher. selır kurz. Die hintere Basalzelle mit der Discoidalzelle verschmolzen, nicht durch eine Querader von ihr getrennt. Kleine, schlanke, meist metallisch glänzende Fliegen. Fam. Dolichopidae (partim), Glanzfliegen.

- Vordere Basalzelle bis gegen die Flügelmitte verlängert, die hintere durch eine Querader von der Discoidalzelle abgetrennt.

Fam. Empisae, (partim) Schnabelfliegen.

16. Analzelle lang, bis zum Flügelrand hin verlängert 17

- Kurz, meist an der Flügelbasis schon abgegrenzt, selten bis gegen die Flügelmitte hin reichend . 19

17. Rüssel hornig, lang, gekniet. Hinterleib ohne Macrochaeten (oder längere Borsten), gestreckt und nach der Bauchseite umgebogen.

Fam. Mropidae.

- Rüssel nicht hornig und nicht doppelt gekniet, oder wenn das in seltenen Fällen vorkommt, so trägt der Hinterleib immer Macrochaeten . . . . . 18

18. Eine vena spuria (d. h. eine nicht von der Flügelwurzel ausgehende, überzählige Längsader) kreuzt die kleine Querader zwischen Ader 3 und 4.

Fam. Syrphidae, Blattlausfliegen.

- Keine die normale Querader kreuzende vena spuria. Augen sehr gross, fast den ganzen Kopf bildend.

Fam. Pipunculidae, Augenfliegen.

19. Hintere Basalzelle mit der Discoidalzelle verschmolzen, die trennende Querader fehlt.

Fam. Dolichopidae (partim).

- Hintere Basalzelle durch eine Querader von der Discoidalzelle getrennt 
20. Rüssel rudimentär, Taster fehlend oder verküumert. Bremsenartige Parasitenflieren von Säugethieren.

Faun. Oestridae, DasselHiegen.

- Rüssel und Taster immer deutlich vorhanden . . . 21

Fam. Muscidae, Gemeinfliegen.

21. Flügelschüppchen deutlich, meist stark entwickelt.

Muscidae calytreae.

- Flügelschüppchen verkümmert oder ganz fehlend.

Muscidae acalyptreae.

\section{Familie Stratiomyidae, Waffenfliegen.}

Die Stratiomyiden im engern Sinne sind bunte, oft sogar metallisch glänzende, schöne Fliegen, die träge auf Blumen herum sitzen. Ihr halbkugeliger, kurzer Kopf ist meist fast so breit als das Brustschild. Die Fühler sind nicht weit rom Mundrand eingefügt, einander genähert, vorgestreckt, mit deutlich geringeltem, cylindrischen Endglied, mit oder ohne Endborste oder Griffel. Scliildchen oft seitlich oder hinten mit kleinen Dornen bewaffnet, Beine relatir schwach, kahl, oline Borsten, Füsse mit drei Haftläppchen (pulvilli). Flügelschüppchen sehr klein, oft felllend. Flügel dem Leib horizontal autliegend und daran leicht kenntlich, dass die 4 vordern Längsadern auffallend stark an den Vorderrand gerückt sind und dicht zusammenliegen, dass Ader I. und II. in einen Ast verschmolzen scheinen, so dass die kleine Discoidalzelle sehr weit nach vorn zu liegen kommt. Die von ihr ausgehenden $3-4$ Längsadern schwach, erreichen den Flügelrand kaum. Die beiden Basalzellen gleich lang, die sie trennende Längsader 4 (cubitus anticus) sehr schwach. Analzelle fast bis zum Flügelrand reichend. Alle Hinterrandzellen offen. Sie lieben die Nähe del Gewässer und Sumpfwiesen. Von vielen leben die Larven im Schlammwasser und verpuppen sich in der Larvenliaut. Mit dieser Familie vereinigen wir hier zwei isolirte Arten, die sonst als Repräsentanten von zwei Fainilien gelten, und deren Flügelgeäder sich dadurch unterscheidet, dass eimmal die vier ersten Längsadern nicht so dicht an den Vorderrand gerückt sind, die grössere Discoidalzelle daher in die Flügelmitte zu liegen kommt, die Randader, wenn auch schwach, den Hinterrand des Flügels umzieht. Das plumpe Genus Coenomya theilt mit den Waffenfliegen den Character eines, mit 2 Dornen bewaffneten, Schildchens und ist daher leicht $\mathrm{zu}$ orkennen. Der schlanke Xylophagus hat kein dẹtlich bedorntes Schildchen, nur an der Seite ist 
es eingedrückt, so dass sein Aussenrand wie ein winziges Dörnchen vorspringt.

\section{Gat tug un der Waffenfliegeu.}

1. Die 4 vordern Längsadern des Flügels dicht an den Vorderrand gedrängt. Discoidalzelle sehr kleiu, nahe an den Vorderiand gerückt, mit $3-4$ schwachen Ausläufern (Stratiomyidae sensu stricto)

- Die 4 vorderen Längsadern nicht dem Vorderand genähert, Discoidalzelle ziemlich gross, in der Flügelmitte mit 3-4 starken, den Flügelrand erreichenden Endästen (Xylophagidae und Coenomyidae) . . . 13

2. Hinterleib mit 4-5 deutlichen Segmenten, Schildchen unbedornt oder nur mit 2 kleinen Dornen .

- Hinterleib mit mindestens 7 Segmente. Schildchen mit 2, 4 oder 6 Dörnchen . . . . . . . . . 11

3. Aus der Discoidalzelle entspringen wur 3 gegen den Flügehand zielıende Längsadern. Sehr kleine schwärzliche Fliegen.

Pachygaster Meigen.

- Aus der Discoidalzelle, oder aus ihr und der wurzelwärts von derselben gelegeneu hintern Basalzelle entspriugen 4 zum Flügelrand gehende Längsadern

4. Schildchen nicht gedornt, Hinterleib meist schmal, langgestreckt. Metallisch grüne Flıegen mit oder ohne lichtere Flecken und Binden

- Schildchen gedornt oder nackt, Hinterleib kurz, eiförmig, nicht metallisch, vorherrschend braun oder schwarz mit liellen Flecken und Binden, oder gelb mit dunkeln Flecken

5. Hinterleib stark verlängert. Fühlerborste nicht ganz endständig, etwas vor der Spitze angebracht, Augen des Jännchens durch die Stirne getrennt. Sargus Fab.

- Hinterleib weniger gestreckt, Fühlerborste ganz endständig, Augen des Männchens an der Stirne zusammen stossend.

Chrysomyia Macq.

6. Schildchen nicht bedornt . . . . . . . . 7

- Schildchen bedornt . . . . . . . . . . . 8

7. Untergesicht schuauzenförmig vorgezogen. Sehr kleiue Fliegen.

Nemotelus Geoffr

- Untergesicht wenigr prominent. Lasiopa Brullé.

8. 'Thorax yor ler Flügelwurzel jederseits mit einem Dorn. Grosse ganz schwarze Fliegen mit brennend rothem Rückenschild; der Kopf viel kleiner als der Thorax.

Ephippium Latr. 
- 'Thorax ohne Dornen. Kopf fast so breit als der Rücken

9. Frstes Fühlerglied $3-4$ mal länger als das zweite. Hinterleib breit, meist mit gelben Seitenflecken. Ueber $1 \mathrm{~cm}$. grosse Fliegen.

Stratiomys Geoffr.

- Frstes Fïhlerglied kaum doppelt so lang, oder bloss so lang als das zweite. Kleinere anders gefärbte Fliegen

10. Endgriffel des letzten Fühlergliedes kurz. Mittelgrosse Fliegen.

Odontomyia Meig.

- Endgriffel des letzten Fühlergliedes lang und dünn. Kleinere Fliegen.

11. Aus der Discoidalzelle entspringen bloss 3 Längsadern. Hinterleib ziemlich gestreckt.

- Ans der Discoidalzelle entspringen 4 Längsadern, von denen indess die dritte oft nur rudimentär und schwach angedeutet ist

12. Abdomen breit, stark gewölbt. Taster undeutlich.

Acanthomyia Schiner.

- Abdomen schmal, seitlich blattartig zusammengedrückt, Taster deutlich dreigliederig. Actina Meig.

13. Grosse dickbänchige braun-gelbe Fliegen mit 2 J)̈̈rnchen am Hinterrande des Schildchens. Ihr Kopf viel schmäler als der Thorax.

Coenomyia Latr.

- Schlanke gestreckte Fliegen, Schildchen ohne Iornen, nul seitlich etwas eingedrückt. Kopf so breit wie der Thorax. Flügel getleckt mit rauchig angelaufener Querader.

Xylopharus Meig.

\section{Familie Tabanidae, Bremsen.}

Die Bremsen sind grosse, kräftige, wenig behaarte Fliegen voll braunschwarzer oder grauer Farbe, deren Weibchen wenigstens das Blut der Menschen und Thiere saugen mit ihren 4-6 klingenartigen Borsten. Die Fïhler des grossen Kopfes sind einander genähert, ihr letztes Glied an der Basis meist erweitert, oft mit einem tiefen Ausschnitt verselıen. Die Stirne des $?$ breit, mit glänzend schwarzen Flecken, Augen gross, im Leben oft mit Purpurbinden durchzogen. Punktangen fehlen den ächten Tabaniclen, während sie bei der Interfamilie der Pangonidae vorhanden sind. Hinterleib der $\subseteq$ eifürmig, der o zugespitzt. Die Randader mmzieht auch den hintern Flügelrand. Alle Hinterrandzellen offen in den Rand mündend. Die Larven leben in fenchter Frde von verwesenden Pflanzenstoffen. 


\section{Gattungeuder Bremseu.}

1. Ohne Punktaugen. Hinterschienen ohne einen Enddorn. Grosse Bremsen (Tabanini) . . . . . . 2

- Mit Punktaugen. Minterschienen mit einem sehr kleinen Enddorn. Mittelgrosse Breınsen . . . . 5

2. Drittes Fühlerglied an der Basis spatelförmig erweitert, oft einen basalen Zahn tragend, fünfringelig 3

- Drittes Fühlerglied gerade, nicht erweitert, nicht ausgeschnitten. duren deutlich behare (Man muss mit starker 3. Augen deutlich behaart. (Nan muss mit starker
Lupe unter schief einfallendem Licht beobachten.)

'Therioplectes Zoller.

- Augen uackt oder undeutlich beliaart.

Tabanus L.

4. Letztes Fühlerglied durch tiefe Einschnitte in 4 deutliche Ringel getheilt, so dass die Fühler scheinbar 6 gliederig sind. Grössere Bremsen. Ilexatoma Meig.

- Letztes Fühlerglied selır undeutlich geringelt, die Fühler daher mur dreigliederig. IIaematopota Meig.

5. Flügel mit schwarzen Binden oder Flecken. Kleinere Stechbromsen.

Chrysops Meig.

- Flügel olne schwarze Binclen, höchstens mit bräun-

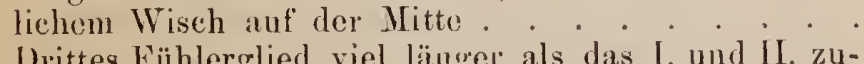
6

6. Drittes Fühlerglied viel länger als das I. und II. zusammen, zwrites so lang als breit. Grössere, hellgelbe Bremsen.

Silvius Meig.

-. Drittes Fühlerglied nur so lang als Glied I. und II. Zweites länger als breit.

Nemorius Rond.

\section{Familie Bombylidae, Schwebfliegen.}

Meist dicht behaarte, mittelgrosse Fliegen mit kleinem, kalbkugeligem Kopf, an dem der hornige Saugrüssel oft weit vorgestreckt ist. P'nnktaugen vorhanden. Fïhler vorgestreckt, mit ungeringreltem Findglied, an der Spitze mit kurzem Griffel oder Borstenbüschel, oder ganz nackt. Beine dünn und schwach, Leib dick. Fügel in der Ruhe abstehend o ler ausgrebreitet, mit einer Discoidalzelle, 2-3 Unterraudzellen und höchstens 4 Hinterrandzellen. Die Analzelle reicht bis zum Flügelrand. Ilı eigenthümlich schwebender Flug hat ilınen den Namen verschafft, da sie oft rüttelnd wie ein fester Pınkt in der Luft stehen bleiben. Sie sind wohl meistens Parasiten von Hymenopteren. 


\section{Gattugeu der Schwobfliegen.}

1. Fühlerbasis von einander entfernt stehend. Stirne in beiden Geschlechtern breit, so dass die Augen des Männchens getrennt sind. Flügel oft schwarz gezeichnet (Anthracinae)

- Fühler an der Basis einander sehr genähert; Augen des $\sigma^{7}$ berühren sich an der Stirne ganz oder fast ganz. Flügel oft mit braunen Zeiclınungen (Bombylinae) .

1. Untergesicht stark vorgezogen. Die zweite Unterrandzelle durch eine Querader entzweigetheilt, so dass 3 Unterrandzellen vorhanden sind.

Exoprosopa Macq.

- Untergesicht rundlich, wenig vorgezogen. Nur 2 Unterrandzellen . . . . . . . . . . . 2

2. Obere und untere Gabelzinke der dritten Längsader (sector Radii) mit einem rücklaufenden Aderstrang. Drittes Fühlerglied kugelig, an der Spitze mit einem kleinen Borstenbüschel.

Argyramoeba Schiner.

- Selten der untere, nie der obere Ast des sector Radii mit einem rüicklaufenden Aderstrang. Drittes Fühlerglied zwiebelförmig oder conisch, mit zweigliedrigem Endgriffel.

Anthrax Scop.

3. Rüssel kırz, wenig vorstehend, mit breiten SaugHächen, wie bei Anthrax.

Lomatia Meig.

- Rüssel sehr lang, hornig, weit und horizontal vorstehend, spitz, mit schmalen Sangflächen . . . . 4

4. Drei Unterrandzellen, indem die hintere durch eine Querader getheilt ist. Erstes Fïhlerglied dick und länger als das zweite, drittes ohne Borstenhaare an Ende.

Ploas Latr.

- Nur zwei Unterrandzellen

5. Vordere Basalzelle länger als die hintere. Erste Hinterrandzelle geschlossen. Das verlängerte dritte Fühlerglied mit einem 3gliedrigen Endgriffel. Augen der ठో zusammenstossend.

Bombylius L.

- Vordere Basalzelle so lang als die hintere. Sonst der vorigen Gattung gleich. Die Augen des c berühren sich nicht ganz.

Systoechus Loew. 


\section{Familie Asilidae, Raubfliegen.}

Meist kräftige, mittelgrosse bis sehr grosse Fliegen mit starken beborsteten Beinen, deren Hinterschienen einen Enddorn, und deren Füsse 2 Haftläppchen haben. Ihr breiter und kurzer Kopf hängt an langem Halsstiel und trägt stark vorquellende Augen, zwischen denen die eingesattelte Stirne tiefer liegt. Rüssel stark, horizontal oder schief vorstehend, mit 4 Stechborsten. Augen bei $\sigma^{7}$ und $\$$ getrennt. 3 Punktaugen, die oft auf einer Erhöhung stehen. Die Genitalien des $\checkmark$ und die Legröhre des $Q$ an dem 8ringeligen Hinterleib meist stark vortretend. Flïgel dem Leib flach aufliegend, mit Discoidalzelle und gegabelter dritter Juängsader, mit 2-3 Unterrandzellen, 4-5 Hinterrandzellen und bis zum Rande reichender Analzelle. Basalzellen ungefähr gleich lang. Die Fliegen sind kecke Räuber, die oft viel grössere Beute iiberfallen, die Larven in feuchter, sandiger Erde, leben von faulen Pflanzenstoffen. An sonnigen Planken und Felsen sitzen die Fliegen auf der Lauer und sind wegen ihres unstäten eigenthümlichen Abfluges schwer zu fangen. Die Puppen sind Mumienpuppen.

\section{Genera der Raubliegen.}

1. Radialzelle (Randzelle) offen in den Flügelvorderrand mündend

- Radialzelle geschlossen und meist gestielt. Nur 3 Hinterrandzellen münden offen in den Flügelrand

2. Füsse ohne Haftläppchen. Aus der Discoidalzelle entspringen 4 zum Flügelrand laufende Adern, die erste in der Mitte zwischen kleiner Querader und dem Ende der Discoidalzelle. Sehr schlanke Arten.

Leptogaster Meig.

- Füsse mit 2 Haftläppchen. Aus der Discoidalzelle 3 Aeste zum Flügelrand gehend, der erste (cubitus anticus) näher dem Ende der Discoidalzelle als der kleinen Querader entspringend.

3. Die 4 Hinterrandzellen münden alle offen in den Flügelrand .

- Nur 3 Hinterrandzellen offen in den Flügelrand mündend, die dritte ist geschlossen oder doch fast geschlossen

4. Drittes Fülllerglied mit zweigliedrigem, stumpfem Endgriffel.

Dioctria Mg.

- Drittes Fühlerglied mit spitzem, undeutlich gegliedertem Endgriffel 
5. Vorderschienen in einen dicken, hackigen Dorn auslaufend.

Dasypogon Mg.

- Vorderschienen ohne Enddorn . . . . . . . . 6

6. Untergesicht mit starkem Höcker, der seine ganze Länge einnimmt.

Cyrtopogon Loew.

- Untergesicht ohne oder nur mit kleinem Höcker . 7

7. Kleine Arten mit verdickten Hinterschienen und Metatarsen.

IIoplopogon Loew.

- Grössere Arten mit einfachen Beinen. Isopogon Loew.

8. Vorderschienen mit dickem Enddoru. Dasypogon Mg.

- Vorderschienen ohne Enddorn . . . . . . . 9

9. Kopf höher als breit. Erste Hinterrandzelle stark verengt.

Stenopogon Loew.

- Kopf breiter als hoch. Erste Hinterrandzelle nicht verengt.

Lasiopogon Loew.

10. Erste Hinterrandzelle geschlossen. Drittes Fühlerglied blattartig, kaum länger als breit, ohne Endborste.

Andrenosoma Rondani.

- Erste Hinterrandzelle offen in den Flügelrand mündend . . . . . . . . . . . . . . 11

11. Drittes Fühlerglied ohne Griffel oder Borsten.

Laphria Mg.

- Drittes Fühlerglied mit einer Endborste 12 Asilus L.

Das Genus Asilus wird von Loew. in eine grosse Anzahl von Subgenera aufgelöst, von denen wir hier folgende weiter als schweizerische anführen:

12. Die weibliche Legröhre seitlich zusammengedrückt 13

- Die weibliche Legröhre nicht zusammengedrückt 21

13. Thorax bis vornhin mit fast gleichlangen Borsten bekleidet.

Lophonotus Loew.

- Thorax nur bis zur Mitte mit langen Borsten bekleidet, vorn mit kleinern und spärlichen Borsten oder kahl oder weichhaarig .. . . . . . . . . . 14

14. Endlamellchen, welche die Wurzel der Legröhre umgeben, eiförmig, in die Legröhre eingekeilt.

Eutolmus Loew.

- Endlamellchen frei, fast griffelförmig

15. Hinterrand des 8 . Segmentes beim or erweitert. . 15

Machimus Loew.

- Hinterrand des 8. Segmentes nicht erweitert beim $\sigma^{\top} 16$ 
16. Beine vorherrschend gelb, glänzend. Mochtherus Loew.

- Beine vorherrschend schwarz oder hell und matt . 17

17. Hinterleib oben und unten glänzend schwarz.

Stilpnogaster Loew.

- Hinterleib nicht glänzend schwarz . . . . • . 18

18. Körper gelbgrau oder aschgrau gefärbt. Beine rothgelb, gestreift oder geringelt.

Epitriptus Loew.

- Körper mehr schwarzgrau gefärbt. Beine vorherrschend schwarz, nur die Schienen bei einigen Arten roth . . . . . . . . . . . . . . . . 19

19. An der Bildung der Legröhre betheiligt sich schon das 6. und 7. Segment, die $\sigma$ Genitalien dickkolbig

Itamus Loew.

- Die Legröhre nicht vom 6. Segment an ausgezogen. Die männlichen Genitalien nicht kolbig . . . 20

20. Männliche Genitalien von oben gesehen am Ende spitz. Untergesichtshöcker stark.

Tolmerus Loew.

- Männliche Genitalien von oben gesehen stumpf. Untergesichtshöcker sehr klein. Cedristus Loew.

21. Hinterleib ohne Borsten (Macrochaeten) vor den Einschnitten

- Hinterleib mit Borsten vor den Einschnitten. . 24

22. Bart feinhaarig niedergedrückt.

Antiphrisson Loew.

- Bart meist rauhhaarig .

23. Hinterleib mit langer, zerstreuter Behaarung.

Pamponerus Loew.

- Hinterleib feinhaarig, Haare niedergedrückt. Grosse bundgefärbte Arten.

Asilus L. Loew.

24. Weibliche Legröhre dick, kolbig, unten dicht und filzig behaart.

- Legröhre konisch, am Ende mit Stachelborsten.

Philonicus Loew.

Fs ist klar, dass diese Subgenera keine bequeme Analyse bilden, weil grossentheils Merkmale benutzt sind, die nur einem Geschlechte entnommen wurden. Auch sind viele Criterien nicht wertlıvoll genug, um wirkliche generische Unterschiede abzugeben, dennoch haben wir die Tabelle zur Tervollständigung hier eingefügt, und sie mag dann als Controle für die Gruppirung und Rectification der zahlreichen Species dienen, die wir alle in das Genus Asilus stellen müssen. 


\section{Die Arten der Waffenfliegen.}

1. Gen. Pachygaster Meig. Kleine, glänzend schwarze Fliegen mit 5eckiger Discoidalzelle, aus der 3 sehr schwache Adern gegen den Flïgelrand abgehen. Larven in faulem Holz.

1. Flügel an der Wurzelhälfte braun. Beine mit schwarzen Schenkeln.

Ater Panz.

- Flügel głashell, Beine ganz gelb.

Leachii Curtis.

2. Gen. Nemotelus Geoffr. Kleine Fliegen mit weissgelben Zeichnungen des Hinterleibes. 4 blasse Adern gehen von der Discoidalzelle $a b$. In der Nähe von Gewässern lebend.

1. Oberseite des Abdomens ganz schwarz, über den Fühlern kein weisser Fleck. Beine schwarz. Kiniee, Schienen und Tarsen gelblich. Nigrinus Curtis.

- Oberseite des Abdomens vorherrschend weissgelb mit schwarzen Flecken. Ueber deı Fühlern ein heller Fleck .

2. Bauch mit Ausnahme der Wurzel ganz weissgelb. Oberseite des Hinterleibes nur an der Wurzel und an der Spitze mit kleiner, schwarzer Mackel. Beim $\sigma$ geht die weisse Zeichnung weiter. Pantherinus L.

- Bauch schwar\% mit gelben Einschnitten und gelbem Mittelflecken an der Wurzel. Oben ein grosser Mittelfleck bis zum 2. Ring reichend.

Uliginosus Loew.

3. Gen. Lasiopa Brullé, villosa F. Mittelgrosse Fliege, schwarz mit 3 Paaren weisser Binden auf dem Hinterleib.

4. Gen. Ephiopium Latr., thoracicum Latr. Eine grosse schwarze Fliege, deren rothglänzend bchaartes Bruststück sofort auffällt. Schildchen mit 2 Dornelı.

5. Gen. Oxycera Meig. Kleinere bis mittelgrosse Fliegen von schwarzer Farbe mit gelben oder grünen Flecken. Schildchen mit 2 Dornen.

1. Hinterleib vorherrschend schwar' mit gelben oder grünen Flecken und Binden

- Grundfarbe des Körpers grüı mit schwarzen Flecken und Binden. Fühler gelb mit gebräunten Spitzen. Stirne des $\$$ mit schwarzer Mittelstrieme. Thorax mit 3 breiten, schwarzen Längsbinden. Abdomen mit 3 schwarzen, den Seitenrand nicht erreichenden Quer- 
flecken. Der Hinterrand der grünen Segmente ist schwarz gesäumt. Halteren grün. $5 \mathrm{~mm}$. Trilineata F.

2. Flügel hyalin, ohne braunes Flügelmahl über der Discoidalzelle

- Flügel am Vorderrand mit braunem Fleck über der kleinen Discoidalzelle. Ganz schwarz bis auf die Halteren und Afterspitze. Beim $Q$ ist auch noch das Schildchen gelblich-weiss. Ebenso sind Kniee, Sichienen und Taster gelblich, die Hinterschienen mit brauner Mitte. 4-5 $\mathrm{mm}$.

Analis Mg.

3. Hinterleib auf den Mittelringen mit gelben Flecken 4

- Hinterleib ganz schwarz mit Ausnahme eines Fleckens an der Wurzel und der Afterspiste. Kopf schwarz. Beim $Q$ am vordern Augenrand silberglänzend, hinterer Augenrand gelb gesäumt. Thorax schwar'z mit schmalem gelben Seitenrand und einem Flecken vor der Flügelbasis. $5 \mathrm{~mm}$.

Leonina $\mathrm{Mg}$.

4. Schildchen ganz gelb

Schildchen bis auf die Dornen schwarz, selten schmal, gelb gesäumt, Thorax oben schwarz mit schmalgelbem Seitenrand und 2 kleinen Flecken an der Flügelwurzel. Abdomem oben auf dem 3. und 4. Ring mit zwei weissen Flecken. $3 \mathrm{~mm}$.

Pygmaea Fall.

5. Beine vorwiegend gelb

- Beine vorwiegend schwarz

6. Schenkel ganz gelb

- Schenkel an der Basis oder in der Mitte schwarz.

7 . Q $Z$ wei schmale gelbe Binden an den Seiten der Augen durchziehen das Untergesicht bis zum Scheitel und convergiren nach oben. Brust schmal, gelbgesäumt, mit zwei schmalen gelben Rückenstreifen. $4 \mathrm{~mm}$.

Pardalina Mg.

Q Zwei breite parallele Kopfbinden über die Gesichtund Scheitelfäche. Brust breit gesäumt mit 2 Längslinien auf der Mitte. $4 \mathrm{~mm}$.

Formosa Meig.

8. Seitenmakeln des Hinterleibes unter sich und von der Endmakel deurlich getrennt, nicht durch lıelle Randbinden vereinigt

- Seitenmakeln des Hinterleibes unter sich und mit dem Afterflecken durch den hellen Leibesrand verbunden

9. Q mit 2 nach oben convergierenden Gesichtsbinden. Brust nit gelbem Saum und 2 Rückenbindeı, 
8 ohne diese letzteren. Beine gelb mit schwarzen Schenkeln und Schienenringen. Fühler schwarz. $5 \mathrm{mim}$.

Amoena Loew.

- Die zwei ersten Fühlerglieder rothbraun. Im übrigen der rorigen Art sehr ähnlich, aber vor der Flügelwurzel auf dem Thorax jederseits ein gelber Fleck, welcher amoena fehlt.

Engadinica Jaennicke.

10. Nur 2 Paar gelbe Seitenflecken und ein Afterfleck am Abdomen. Thorax sehr breit gelb gesäumt. Die gelben Hinterleibsmakeln gehen schief nach innen. $6-7 \mathrm{~min}$.

Pulchella.

- Drei Paar Seitenflecken am Abdomell. Die gelben Zeichnungen auf dem Thorax vorn mit den Seitenmakeln zu halbmondförmigen Bogen rerbunden. Fühler braungolb. $8 \mathrm{~mm}$.

Meigenii Staeger $\mathrm{F}$.

11. Beine schwarz mit gelben Knioen und Schienenspitzen. Das letzte gelbe Fleckenpaar am Abdomen nach innen erweitert, daher in der Mitte viel $m$ ehr genähert, als die vorhergehenden Pare. $6-\$ \mathrm{~mm}$.

Locuples Loew.

6. Stratiomys Geoffr. Grössere, $15 \mathrm{~cm}$. grosse, breite Fliegen mit gelben Flecken-Zeichnungen auf dem Hinterleib. Erstes Fühlerglied selır lang, drittes schmal, fünfringelig und winkelig abstehend. Augen des $\sigma$ fast zusammenstossend mit kleinern Fascetten auf der Unterseite. Beim ? ist der Hinterrand der Augen meist gelb. Schilichen mit 2 Dornen. Die Fliegen sitzen träge auf Blumen, die Larven leben im Schlammwasser.

1. Abdomen oben mit gelben Seitenmakeln oder mit Binden rersehen.

- Abdomen ohne gelbe Binden, höchstens mit lichterer Behaarung an den Seiten. Schwarz, dicht belıart.

Longicornis Scop.

2. Hinterleib oben mit 3 Paar getrennter gelber Seitenmakeln .

- Hinterleib nur mit 2 Paar Seitenmakeln und an 4. Ring eine durchgehende gelbe Hinterraudbinde, oder nur 2 Paar Makeln und 2 gelbe Hinterrandbinden am 3. und 4. Ring. Bauch gelb mit 4 schwarzen Querbinden.

Potamida Mg.

3. Bauch vorwiegend seliwarz, mit hellen Hinterrandsäumen oder Querflecken

- Bauch rorwiegend gelb, mit schwarzen lilecken . 7 
4. Eühler schwarz. Rückenschild rothgelb oder grau behaart

- Fühler rothbraun bis schwarzbraun. Rücken schwarz behaart. Ueber den Fühlern ein gelber Fleck. Das erste Fleckenpaar auf dem Abdomen breit, die andern schmal, bindenf örmig.

Concinua Mg.

5. Erstes Fühlerglied nur halb so lang als das dritte. Q mit gelben Seiten des Untergesichtes; die gelbe Farbe setzt sich bis über die Fühler fort und bildet dort 2 halbmondförmige Flecke. Augenrand des ? gelb. Schildchen beim ơ schwarz, nur die Spitze und die Zähnchen gelb. Seitenflecke am Abdomen des ó sehr schmal, bindenförmig.

- Erstes Fühlerglied ungefähr so lang als das dritte. Q ohne gelbe Begrenzung des Untergesichtes. Ueber jedem Fühler und jedem Auge ein gelber Fleck.

$\mathrm{Mg}$

. Oberseite des Hinterleibes mit ziemlich breiten gelben Seitenflecken.

Furcata F.

- Oberseite des Hinterleibes mit weisslichen Seitenstrichen und gleichfarbigem After.

Riparia $\mathrm{Mg}$.

7. Spitze des dritten Fühlergliedes stumpf . . . . 8

- Spitze des dritten Fühlergliedes unregelmässig in drei ungleiche Zipfelchen gespalten, sonst wie Chamaeleon.

Unguicornis Becker.

8. Schildchen gelb mit abgerundetem schwarzen Basalfleck.

Cenisia Mg.

- Schildchen gelb mit dreieckigem schwarzen Basalfleck .

9. Beine gelbroth, Schenkel des $\sigma^{\prime}$ schwarz, Schienen in der Mitte mit schwarzem Ring. Beim $\&$ nur die Spitzenhälfte der Schenkel schwarz. Mundrand des O breit schwarz gesäumt. Die schwarzen Bauchbinden beim $\sigma^{\pi}$ und $\odot$ gleich stark ausgeprägt.

Rhaetica Jaennicke.

- Beine gelb, beim $\sigma^{7}$ mit schwarzen Schenkeln und schwarzen Schienenringen, beim $ᄋ$ mit schwarzen Schenkelringen und schwarzen Schienenspitzen. Die Rückenflecke des Hinterleibes spitzig nach innen zu. Die schwarzen Bauchflecke liegen auf der Mitte des 2.-5. Segmentes und sind beim $q$ grösser als beim $\sigma^{7}$

Chamaeleon De Geer.

7. Odontomyia Meig. Mittelgrosse bis kleinere, schwarze Fliegen mit gelben oder grünen Zeichnungen auf dem Hinter- 
leib. Fühler mässig lang, Endglied vierringelig mit zweigliederigem Endgriffel. Augen des $\sigma^{7}$ zusammenstossend. Der vorstehende Rüssel mit breiten Saugflächen. Leben wie Stratiomys-Arten.

1. Grosse Art von ca. $15 \mathrm{~mm}$. und rom Habitus einer Stratiomys. Hinterleib oben mit 3 Paaren gelber, fast gleich grosser Seitenflecke. Fühler braungelb.

Ornata Mg.

- Kleinere Arten von höchstens $10 \mathrm{~mm}$. Grösse, 1 it anderer Zeichnung und Färbung . . . . . . . 2

2. Erstes Fühlerglied so lang als das zweite . . . 5

- Erstes Fühlerglied doppelt so lang als das zweite . 4

4. Goldgelb bis braungelb behaart, an dem Hinterleib kleine gelbe Seitenflecken und am Hinterrand der Segmente goldglänzende Haarbinden, Schildchen dicht gelb behaart. Untergesicht gelb. $8 \mathrm{~mm}$. Argentata $\mathrm{F}$.

- Wenig behaart. Schildchen schwarz mit kleinen gelben Hörnchen. Drei Paare schmaler gelber Halbbinden am Hinterrand des 2., 3. und 4. Segmentes, ohne goldglänzende Haarbinden. Stirn schwarz, beim o mit 2 kleinen gelben Stirnflecken; Untergesicht glänzend schwarz.

Vicroleon $\mathrm{L}$.

5. Seitenrand des Hinterleibes grün oder grüngelb gefleckt oder gerandet

- Seitenrand des Hinterleibes breit grelb gesäumt, so dass die Mitte von einer etwas ausgebuchteten schwarzen Längsstrieme durchzogen ist. Bauch orangeroth. Schenkel schwarz mit gelben Ḱnieen, Hinterschienen schwarz geringelt. $10 \mathrm{~mm}$.

Flarissima Rossi.

6. Discoidalzelle mässig gross mit 2 deutlichen Längsadern

- Discoidalzelle sehr klein, gibt nur eine deutliche Längsader ab, die erste und dritte obliterirt. Die schwarze Längsbinde a uf dem Rücken des Hinterleibes in Breite sehr variabel, aber hinten stets breiter als in der Mitte. Untelgesicht spitz vorspringend. Thorax und Schildchen schwarz, beim $q$ goldharig glänzend. Beine gelb, nur die Hüften schwarz. $5-8 \mathrm{~mm}$.

Viridula F.

7. Die schwarzen Rückenflecken auf dem Hinterleib fast gleich gross, nicht bindenartig erweitert. \ ohne schwarze Flecken am Untergesicht. $10 \mathrm{~mm}$. 
- Die schwarzen Rückenflecken auf dem Hinterleib rorn bindenartig erweitert, besonders auf dem 3 . und 4. Segment. $\&$ mit 2 deutlichen schwarzen Flecken auf dem gelben Untergesicht

8. Beine ganz gelb. $.10 \mathrm{~mm}$.

- Schenkel schwarz, gegen die Spitzen zu gelblich.

IIeydeni Jaennicke.

8. Sargus F. Metallisch glänzende, schlanke Fliegen mit langgestrecktem Hinterleib. Letztes Fühlerglied rundlich, vierringelig mit zweigliedriger Borste. Stirne in beiden Geschlechtern ziemlich breit. Schildchen ohne Dornen. $\sigma$ mit stumpfem, nach hinten verbreiterten Hinterleib, ᄋ mit zugespitztem Hinterleib, der hinter der Mitte am breitesten ist. Lebhafte Fliegen, auf Blättern sitzend, seltener auf Blumen. Die Larven in Dung und Mulm.

1. Beine ganz gelb; Punktaugen in ein gleichschenkliges Dreieck gestellt. Hinterleib des $\$$ metallisch blaugrün mit gelbiother Basis, beim ơ erzbraun, einfarbig. $15 \mathrm{~mm}$.

Bipunctatus Scop.

- Beine vorherrschend schwarz. Punktaugen in ein spitzwinkliges Dreieck gestellt. Hinterleib metallisch glänzend, einfarbig . . . . . . . . . . . . 2

2: Beine ganz schwarz oder mit bräunlichen Knieen. Etwa $10 \mathrm{mim}$.

Infuscatus $\mathrm{Mg}$.

- Beine mit gelben Knieen. Etwas kleinere Arten . 3

3. Tarsen der Minterbeine an der Basis und die Kniee deutlich gelb. Flügel mit dunkelm Randmahl auf der Mitte.

Cuprarius L.

- Tarsen der Hinterbeine nie gelb, nur die Kniee gelb. (Wohl nur Varietät der vorigen Art.) Nubeculosus Zett.

9. Chrysomyia Macquart. Kleine bis mittelgrosse metallisch glänzende Arten vom Habitus der vorigen. Augen des $\sigma$ auf der Stirn zusammenstossend. Ohne Dornen am Schildchen. Larven unter Steinen in feuchter Erde.

1. Kleine, kaum $5 \mathrm{~mm}$. messende Art mit nackten Augen. Glänzend goldgrün oder blaugrün. Die breite Stirn des $O$ ist stahlblau. Beine gelb, nur die Schenkel und Mitte der Sclienen schwarz.

Polita L.

- Grössere und schlankere Arten von Sargus ähnlichem Habitus. Ca. $8 \mathrm{~mm}$. Augen behaart. . . . . . 2

2. Fühler gelb, Flügel hyalin, Stirn breit, goldgrün. Goldgrün mit blauem Schiller. Hinterschenkel schwarz, Wurzel und Spitze gelb.

Flavicornis Mg. 
- Fühler schwarz . . . . . . . . . . 3

3. Behaarung des Kopfes gelbbraun, Hintertarsen schwarzbraun und Beine schwarz mit gelben Knieen.

Formosa Scop.

- Behaarung des Kopfes schwarz, Hintertarsen gelblich. (Wohl nur Varietät der vorigen.)

Melampogon Zeller.

10. Acanthomyia Schiner. Einzige Art dubia Zett. Pechschwarze mittelgrosse Fliege mit ziemlich langen Fühlern, drittes Glied achtringelig zugespitzt, die letzten Ringel stiletförmig. Schildchen bedornt, mit 4-6 Stacheln.

11. Beris Latr. Kleine Fliegen von dunkler Farbe und oft gelbrothem Hinterleib. Fühler lang, Endglied schmal, achtringelig. Augen behaart. Schildchen mit 4-8 Dornen. Genitalien des $\sigma^{\text {r }}$ etwas vortretend.

1. Hinterleib gelbroth . . . . . . . . . . . . 2

- Hinterleib dunkelbraun oder schwarz metallisch . 3

2. Hinterleib oben ganz rothgelb, ohne dunkle Quereindrücke. $6 \mathrm{~mm}$.

- Auf dem Hinterrand der sammtartig, gelbrothen Ringel je ein schwarzer Quereindruck. Hinterer Metatarsus des $\sigma$ wie beim vorigen stark verdickt. $6 \mathrm{~mm}$.

Clavipes L.

3. Augen auf der Stirne zusammenstossend, beim $\sigma^{7}$, beim O nur durch eine ganz schmale Leiste getrennt. Fühler länger als der Kopf, an der Spitze mit feinen Börstchen. Hinterleib braunviolett schimmernd.

Morrisii Dale.

- Augren durch eine breite Stirnpartie getremnt 4

4. Hinterleib matt schwarz. Beim o ist der hintere Metatarsus sehr dick, nicht länger als die 4 übrigen Glieder. Beine gelbbraun, ohne braune Querbinden vor der Spitze der Hinterschenkel.

Chalybeata Förster.

- Hinterleib schwarz. Beim $\sigma^{7}$ ist der Metatarsus der Hinterschenkel mässig dick, länger als die 4 übrigen Glieder. Hinterschenkel vor der Spitze mit einem braunen Bändchen.

Fuscipes Meig.

12. Actina Meig. Einzige hiesige Art nitens Latr. Eine kleine metallisch schimmernde Fliege mit einfarbig schwarzem Hinterleib. ठblau schwarz, @ grün. Augen behaart. Schildchen mit schwarzen Dornen. Beine schwarz, $ᄋ$ mit weissen Schenkelwurzelu. 
13. Xylophagus Meig., ater F. Grössere schlanke Fliegen. Die Xylophagiden bilden eine den Waffenfliegen nahestehende Familie, mit unbedorntem Schildchen, aber vom Habitus von Sargus. Kopf so breit als das Brustschild. Die den Vorderrand stützende Randader zieht sich (allerdings verdünnt) auch am Hinterrand hin. Larven im faulen Holz.

14. Coenomyia Latr., ferruginea Meig. Grosse, bremsenartige Fliege, die wir wegen des bedornten Schildchens ebenfalls hierher ziehen. Kopf klein, viel schmäler als der Thorax, dunkelbraun rostgelb. Letztes Fühlerglied undeutlich geringelt. Randader den Hinterrand der Flügel umziehend, daher eine eigene Familie bildend (Coenomyidae). Larven im faulen Holz. 
